\title{
$\sigma-1$ receptor stimulation protects against pressure- induced damage through InsR-MAPK signaling in human trabecular meshwork cells
}

\author{
BO MENG ${ }^{1}$, HONGYI LI ${ }^{2}$, XIAN SUN ${ }^{3}$, WEI QU ${ }^{1}$, BINBIN YANG $^{1}$, \\ FANG CHENG $^{1}$, LIPING SHI ${ }^{1}$ and HUIPING YUAN ${ }^{1}$
}

\author{
${ }^{1}$ Department of Ophthalmology, The Second Affiliated Hospital of Harbin Medical University, Harbin, \\ Heilongjiang 150086; ${ }^{2}$ Department of Ophthalmology and Otorhinolaryngology, Hospital of Heilongjiang University, \\ Harbin, Heilongjiang 150080; ${ }^{3}$ Department of Oncology, The Third Affiliated Hospital \\ of Harbin Medical University, Harbin, Heilongjiang 150081, P.R. China
}

Received January 23, 2016; Accepted March 21, 2017

DOI: $10.3892 / \mathrm{mmr} .2017 .6647$

\begin{abstract}
The purpose of the present study was to investigate the protective effect of the $\sigma-1$ receptor (Sig-1R) agonist (+)-pentazocin (PTZ) on pressure-induced apoptosis and death of human trabecular meshwork cells (hTMCs). The expression levels of Sig-1R and insulin receptor (InsR) were examined in hTMCs. Cells were cultured under a pressure of 0, 20, 40, 60 and $80 \mathrm{mmHg}$ for $48 \mathrm{~h}$, and under $80 \mathrm{mmHg}$ for $44 \mathrm{~h}$, after which the cells were treated with (+)-PTZ $(20 \mu \mathrm{M})$, $\mathrm{N}$-(2-(3,4-dichlorophenyl)ethyl)-N-methyl-2-(dimethylamino) ethylamine (BD-1063; $20 \mu \mathrm{M}$ ) administered $30 \mathrm{~min}$ prior to (+)-PTZ, or BD-1063 (20 $\mu \mathrm{M})$ and then exposed to $80 \mathrm{mmHg}$ again until the $48 \mathrm{~h}$ time-point. The changes of the cells were observed by optical and electron microscopy, the apoptosis and death of hTMCs were detected by ethidium bromide/acridine orange dual staining assay and the expression of Sig-1R and InsR by reverse transcription-quantitative polymerase chain reaction and western blot analysis. The phosphorylation of extracellular signal-regulated kinase (ERK), an important downstream protein of the InsR-mitogen-activated protein kinases (MAPK) signaling pathway, was also detected by western blot analysis when (+)-PTZ and BD-1063 were added to the $80 \mathrm{mmHg}$-treated cells. Sig-1Rs and InsRs were expressed in hTMCs. The apoptosis and death of hTMCs increased from $40 \mathrm{mmHg}$ with $50 \%$ cell death when the pressure was at $80 \mathrm{mmHg}$ and the structure of the cells noticeably changed. The expression of Sig-1R and InsR increased along with the elevation of pressure. (+)-PTZ
\end{abstract}

Correspondence to: Dr Huiping Yuan, Department of Ophthalmology, The Second Affiliated Hospital of Harbin Medical University, 246 Xuefu Road, Harbin, Heilongjiang 150086, P.R. China

E-mail: yuanhp2013@126.com

Key words: primary open angle glaucoma, human trabecular meshwork cells, $\sigma-1$ receptor, insulin receptor decreased the apoptosis and death of hTMCs and increased the expression of Sig-1R and InsR, and the phosphorylation of ERK. Such effects were blocked by BD-1063. The present study suggested that Sig-1R agonist (+)-PTZ can protect hTMCs from pressure-induced apoptosis and death by activating InsR and the MAPK signal pathway.

\section{Introduction}

Primary open angle glaucoma (POAG) is a progressive optic neuropathy often associated with an abnormal elevation of intraocular pressure (IOP) and progressive death of retinal ganglion cells (RGCs) $(1,2)$. The major risk factor for the elevation of IOP is the resistance of aqueous humor (AH) outflow through the trabecular meshwork (TM) (3). On this basis, the preferable treatment for POAG is essentially aimed at maintaining TM function, lowering IOP and protecting RGCs.

Large population-based prevalence and incidence studies (4-6) have identified a positive association between diabetes and POAG. Sato and Roy reported that high glucose levels in the $\mathrm{AH}$ of patients with diabetes may increase fibronectin syntheses and accumulation in TM, and accelerate the depletion of TM cells (7). Basic studies (8-11) have also demonstrated that diabetes, not only affects vascular tissues, but also compromises neuronal and glial functions and metabolism in the retina, which ultimately leads to the apoptotic death of retinal neurons including RGCs. This impaired metabolism of neurons and glial by diabetes may render RGCs susceptible to additional stresses including elevated IOP (8). Although there have been a number of explanations (12-15) for this association, more evidence is required to confirm how diabetes influences IOP and glaucoma. In view of this, a previous study detected the change of the insulin receptors (InsRs) in the high air-pressure cell model employed by this current study (16). Insulin works by binding to the cell surface receptor InsR. There are two main intracellular pathways that are activated by InsRs: The InsR substrate (IRS)-phosphatidylinositol 3-kinase (PI3K) pathway and the Ras-mitogen-activated protein kinase (MAPK) pathway (17-19). 
The $\sigma-1$ receptor (Sig-1R), which has been studied thoroughly in the central nervous system (CNS), is also recognized to be overabundant in the eye, including the lacrimal glands, retina, iris-ciliary body, cornea and lens (20). Modulating Sig-1R activity can lower IOP and protect RGCs (21-23). Sig-1R is a $26-\mathrm{kDa}$ protein that is categorized as a unique non-opioid receptor. It is located at the endoplasmic reticulum (ER)-mitochondrion membrane (MAM) (24) and is a modulator of a variety of receptors and ion channels, acting as an amplifier in signal transduction cascades (25). It functions by binding to various types of exogenous and endogenous ligands, including (+)-pentazocin (PTZ), pregnenolone and N'-[2-(3,4-dichlorophenyl) ethyl]-N,N,N'-trimethylethane-1,2-diamine (26).

A previous study demonstrated that a Sig-1R agonist decreased IOP and protected against retinal damage in a rat model of chronic ocular hypertension glaucoma (27). Nevertheless, the mechanism of this IOP-lowering effect in vitro remains to be elucidated. It is assumed that this effect may be mediated by the increasing outflow of the AH through TM. In the present study, human trabecular meshwork cells (hTMCs) were cultured under different air pressures and Sig-1R agonist (+)-PTZ and antagonist N-(2-(3,4-dichlorophenyl)ethyl)-N-meth yl-2-(dimethylamino) ethylamine (BD-1063) were administered to the cells at high pressure. It was identified that (+)-PTZ can protect hTMCs from pressure-induced apoptosis and death and that the protection was associated with the activation of InsR and its MAPK pathway. The effects of Sig-1Rs on IOP lowering and RGCs protection may make it an efficient therapy for POAG.

\section{Materials and methods}

Drugs. The (+)-PTZ(Sigma-Aldrich; Merck KGaA, Darmstadt, Germany) was dissolved in warm $0.1 \mathrm{~N} \mathrm{HCl}$ and then diluted with pH 7.0 Dulbecco's modified Eagle's medium (DMEM); the BD-1063 (Tocris Cookson Inc., Ellisville, MO, USA) was dissolved in distilled water and then diluted with DMEM.

Cell culture. Cells from the immortalized hTMC line were kindly provided as a gift by the State Key Laboratory of Ophthalmology, Zhongshan Ophthalmic Center of Sun Yat-sen University (Guangzhou, Guangdong, China). The cells were grown in culture flasks in Dulbecco's modified Eagle's medium with $100 \mu / \mathrm{ml}$ penicillin/streptomycin (Hyclone; GE Healthcare Life Sciences, Logan, UT, USA), 20 mM/1 HEPES buffer and $10 \%$ fetal bovine serum (GE Healthcare Life Sciences) in an atmosphere containing $5 \% \mathrm{CO}_{2}$ and $95 \%$ air at $37^{\circ} \mathrm{C}$. The cells were passaged every 3 days using $0.125 \%$ trypsinogen and $0.02 \%$ EDTA buffer (Beyotime Institute of Biotechnology, Haimen, China).

To study the effect of pressure, the cells were cultured in the same conditions as above and the well-grown $50 \%$ confluent cells placed in culture flasks or clusters into the pressure equipment. The cells were subjected to $0,20,40,60$ and $80 \mathrm{mmHg}$ air pressure respectively for $48 \mathrm{~h}$. To study the effect of (+)-PTZ and BD-1063, the cells were cultured under $80 \mathrm{mmHg}$ pressure for $44 \mathrm{~h}$, after which the cells were treated with (+)-PTZ $(20 \mu \mathrm{M})$, BD-1063 $(20 \mu \mathrm{M})$ administered $30 \mathrm{~min}$ prior to (+)-PTZ, or BD-1063 $(20 \mu \mathrm{M})$ and then exposed to $80 \mathrm{mmHg}$ again until the $48 \mathrm{~h}$ time-point.
Pressure equipment. The pressure equipment used in this experiment was designed by the authors and their colleagues and manufactured by a teaching equipment company (Yuying Teaching Device Co., Harbin, China). The equipment comprised an airtight box, a homeothermic incubator (Boxun Medical Biological Instrument Corp., Shanghai, China), a mixed-gas (5\% $\mathrm{CO}_{2}$ and $95 \%$ air) cylinder (Liming Gas Group Co. Ltd., Harbin, China) and rubber tubes. The airtight box was made from plexiglass, which can withstand pressures of 0-120 mmHg. A dial manometer was connected to the top wall and two switch valves to the sidewall of the airtight box; the upper valve was the gas outlet and the lower was the gas inlet, which was connected to the cylinder by rubber tubes. The culture flasks or clusters were placed in the airtight box, the door and the gas outlet closed, then the gas inlet and the cylinder gate opened. When the correct pressure was read from the manometer, the gas inlet and the cylinder gate was closed, to maintain the pressure. Then the homeothermic incubator was set to $37^{\circ} \mathrm{C}$. The atmosphere was renewed every hour to balance the $\mathrm{pH}$ value and the gas concentration in the box.

Ethidium bromide/acridine orange (EB/AO) dual-staining assay. An EB/AO dual-staining kit (Nanjing Keygen Biotech. Co. Ltd., Nanjing, China) was used to assess apoptosis and death of the cells which were cultured under the pressure of 0 , 20, 40, 60 and $80 \mathrm{mmHg}$ for $48 \mathrm{~h}$ respectively, $80 \mathrm{mmHg}$ plus (+)-PTZ $(20 \mu \mathrm{M})$ at $44 \mathrm{~h}, 80 \mathrm{mmHg}$ plus BD-1063 $(20 \mu \mathrm{M})$ $30 \mathrm{~min}$ prior to (+)-PTZ at $44 \mathrm{~h}$ and $80 \mathrm{mmHg}$ plus BD-1063 $(20 \mu \mathrm{M})$ at $44 \mathrm{~h}$. AO can permeate through an unbroken cell membrane and exhibits green fluorescence, while EB can only permeate through a broken cell membrane and exhibits orange-red fluorescence. Normal cells stain green, late apoptotic and dead cells (broken membranes) stain orange-red. Cells were dissociated using $0.125 \%$ trypsinogen and washed twice with $1 \mathrm{X}$ PBS, then incubated with a 5\% EB and 5\% AO mixture for $5 \mathrm{~min}$ at room temperature. The cells were then placed onto slides and visualized using a fluorescence microscope (Olympus BX41; Olympus Corporation, Tokyo, Japan). The number of EB- and AO-stained cells were counted and expressed as the ratio of orange-red cells to total cells for late apoptosis and death rate and then averaged over three different fields.

Transmission electron microscopy of hTMCs. Cells cultured under the pressure of 0 and $80 \mathrm{mmHg}$ were respectively dissociated by $0.125 \%$ trypsinogen, centrifuged at $1,800 \mathrm{x} \mathrm{g}$ for $5 \mathrm{~min}$ at $4^{\circ} \mathrm{C}$ and fixed with $2 \%$ glutaraldehyde in phosphate buffer overnight at $4^{\circ} \mathrm{C}$. Following post-fixation with $1 \% \mathrm{OsO}_{4}$ in cacodylate buffer for $1 \mathrm{~h}$ at $4^{\circ} \mathrm{C}$, the pellet was dehydrated in graded ethanol solutions and embedded in Epon. Ultrathin sections $(80 \mathrm{~nm})$ of pellet were counterstained with uranyl acetate and lead citrate and observed under a transmission electron microscope (JEM1220; JEOL Ltd., Tokyo, Japan).

Reverse transcription-quantitative polymerase chain reaction $(R T-q P C R)$ analysis of Sig-1R and Ins $R$ in experimental $h T M C s$. Experiments were performed to detect the mRNA expression of Sig-1R and InsR in hTMCs which had been cultured under the pressure of $0,20,40,60$ and $80 \mathrm{mmHg}$, and the effects of agonist and antagonist on Sig-1R and InsR 
expression under the pressure of $80 \mathrm{mmHg}$. Total RNA was isolated using the TRIzol reagent (Invitrogen; Thermo Fisher Scientific, Inc., Waltham, MA, USA). cDNA was reverse transcribed from $1 \mu \mathrm{g}$ total RNA in $20 \mu \mathrm{l}$ RT reaction mix using the manufacturer's protocol (Promega Corporation, Madison, WI, USA). Primers and Taqman probes (Shanghai Chaoshi Biotechnology Co. Ltd., Shanghai, China) for human Sig-1R (NM_005866.2) were 5'-AGCTCACCACCT ACCTCTTTGG-3' (forward primer), 5'-ACATGGGCTCCA GCAAGTG-3' (reverse primer) and 5'-FAM-CCTTGACCA GCCAGGCCTGAAGG-BHQ1 (Probe); for human InsR (NM_000208.2) were 5'-GCAGGAGCGTCATCAGCATA-3' (forward primer), 5'-TCCACCCACTGTGAAGGAGAG-3' (reverse primer) and 5'-FAM-TAAATGGATGTGCTGTAG TCCCAGTGCT-BHQ1 (Probe); for internal control human $\beta$-actin (NM_001101.3) were 5'-CCCAGCACAATGAAG ATCAAGATCAT-3' (forward primer), 5'-ATCTGCTGGAAG GTGGACAGCGA-3' (reverse primer) and 5'-FAM-TGACAA GTACTCCGTGTGGATCGGCG-BHQ1 (Probe); qPCR was performed in an ABI Prism 7900 DNA Detection System (Applied Biosystems; Thermo Fisher Scientific, Inc.). Cycling variables were: $2 \mathrm{~min}$ at $50^{\circ} \mathrm{C}, 10 \mathrm{~min}$ at $95^{\circ} \mathrm{C}$ and then 40 cycles of $30 \mathrm{sec}$ at $95^{\circ} \mathrm{C}$ and $30 \mathrm{sec}$ at $55^{\circ} \mathrm{C}$. Cycle threshold values of Sig-1R and InsR were normalized to $\beta$-actin for each sample and calculated by comparison of $2^{\Delta \Delta \mathrm{Cq}}(28)$.

Western blot analysis of Sig-1R, InsR and phosphorylated and total $(p / t)$ ERK in experimental hTMCs. Experiments were performed to detect the protein expression of Sig-1R and InsR in hTMCs which had been cultured under the pressure of $0,20,40,60$ and $80 \mathrm{mmHg}$, and the effects of agonist and antagonist on Sig-1R, InsR and p/t ERK expression under the pressure of $80 \mathrm{mmHg}$. Cells were lysed on ice in RIPA lysis buffer (Beyotime Institute of Biotechnology) plus 1 protease and 1 phosphatase inhibitor cocktail tablets (Complete and PhosSTOP; Roche Applied Science, Penzberg, Germany) per $10 \mathrm{ml}$ lysis. Lysates were centrifuged at 15,000 x g for $5 \mathrm{~min}$ at $4{ }^{\circ} \mathrm{C}$. The protein content of each sample was determined by a spectrophotometer (SmartSpec 3000; Bio-Rad Laboratories, Inc., Hercules, CA, USA). Equivalent amounts of protein from each sample was boiled with protein loading buffer at $95^{\circ} \mathrm{C}$ for $5 \mathrm{~min}$, loaded onto SDS-PAGE gels for electrophoresis and then transferred onto nitrocellulose membrane with a semi-dry transfer cell (Trans-Blot; Bio-Rad Laboratories, Inc.). Membranes were blocked with 5\% skimmed milk for $1 \mathrm{~h}$, incubated with anti-Sig-1R monoclonal antibody (cat. no. sc-166392; 1:100), anti-InsR polyclonal antibody (cat. no. sc-710; 1:200), anti-pERK polyclonal antibody (cat. no. sc-16982R; 1:200), anti-tERK monoclonal antibody (cat. no. sc-514302; 1:200; all Santa Cruz Biotechnology Inc., Dallas, TX, USA) and anti-GAPDH polyclonal antibody (cat. no. KC-5G4; 1:1,000; Kangcheng Biotechnology Co., Ltd., Shanghai, China) respectively at $4^{\circ} \mathrm{C}$ overnight, and GAPDH was used as an internal control. The membranes were then incubated with horseradish peroxidase-conjugated anti-mouse or anti-rabbit IgG antibodies (cat. nos. ZB-2301 and ZB-2305; 1:5,000; Beijing Zhongshan Goldenbridge Biotechnology; OriGene Technologies, Inc., Beijing, China) as the secondary antibodies for $1 \mathrm{~h}$ at $37^{\circ} \mathrm{C}$. Proteins were visualized using a electrochemiluminescence Detection Reagent (cat. no. DQ111-01;
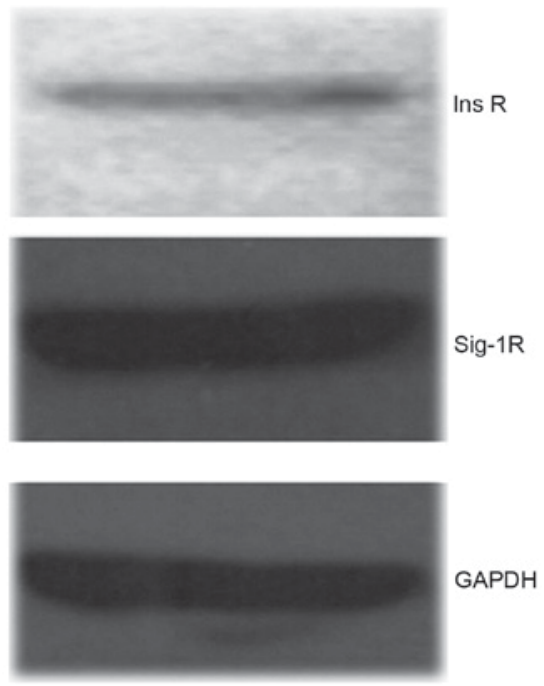

Figure 1. Expression of InsR and Sig-1R in hTMCs. Western blotting of hTMCs demonstrated moderate expression of InsR $(130 \mathrm{kDa})$ and Sig-1R (28 kDa). GAPDH (34 kDa) acted as an internal control. InsR, insulin receptor; Sig-1R, o-1 receptor; hTMCs, human trabecular meshwork cells.

Beijing Transgen Biotech Co., Ltd., Beijing, China), according to the manufacturer's instructions, and captured with a scanner (Epson V30, Seiko Epson Corporation, Tokyo, Japan). Data were quantified using Quantity One version 4.6.2 (Bio-Rad Laboratories, Inc., Hercules, CA, USA) and expressed as the relative value of band density of Sig-1R/GAPDH,InsR/GAPDH and $\mathrm{p} / \mathrm{t} \mathrm{ERK} 1 / 2$.

Statistical analysis. Statistical analyses were performed with the SAS statistical package for Microsoft Windows version 9.1.3 (SAS Institute, Cary, NC, USA). Data were expressed as mean \pm standard deviation for all the measurements. Analysis of variance was used for comparison of experimental groups with control group in EB/AO dual staining assay, RT-qPCR and western blot analysis. The q-test was the post hoc statistical test. $\mathrm{P}<0.05$ was considered to indicate a statistically significant difference.

\section{Results}

Expression of Sig-1R and InsR in hTMCs. RT-qPCR and western blot analysis were used to detect the expression of Sig-1R and InsR and demonstrated moderate amounts of Sig-1R and InsR in hTMCs (Fig. 1).

Pressure-induced abnormalities of hTMCs. Pressure-induced changes in hTMCs were significant. The EB/AO staining assay demonstrated that treatment with pressure increased the number of EB-positive cells considerably, and the rates of EB-positive cells to total cells of the former five groups (cells cultured under $0,20,40,60$ or $80 \mathrm{mmHg}$ of pressure for $48 \mathrm{~h}$, respectively) were $2.63 \pm 1.25,2.80 \pm 1.25,24.50 \pm 2.78$, $39.17 \pm 1.76$ and $57.67 \pm 1.04(\mathrm{P}<0.0001$ with the exception of the 0 and $20 \mathrm{mmHg}$ groups, Fig. 2Aa-e). (+)-PTZ reduced the pressure-induced cell apoptosis and death $(36.00 \pm 2.16$, $\mathrm{P}<0.0001$; Fig. 2A-f) compared with the $80 \mathrm{mmHg}$-treated cells without (+)-PTZ. To confirm that cell protection was 
A a

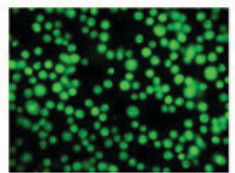

e

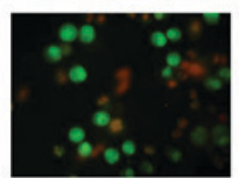

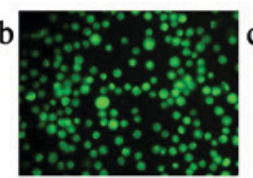

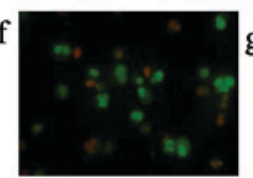

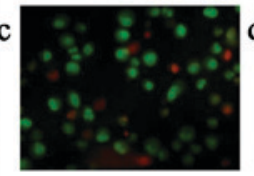

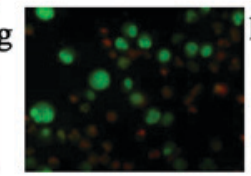

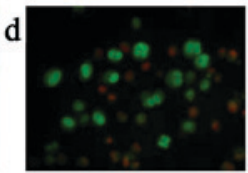

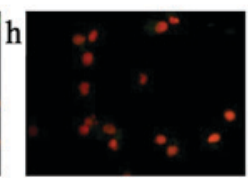

B

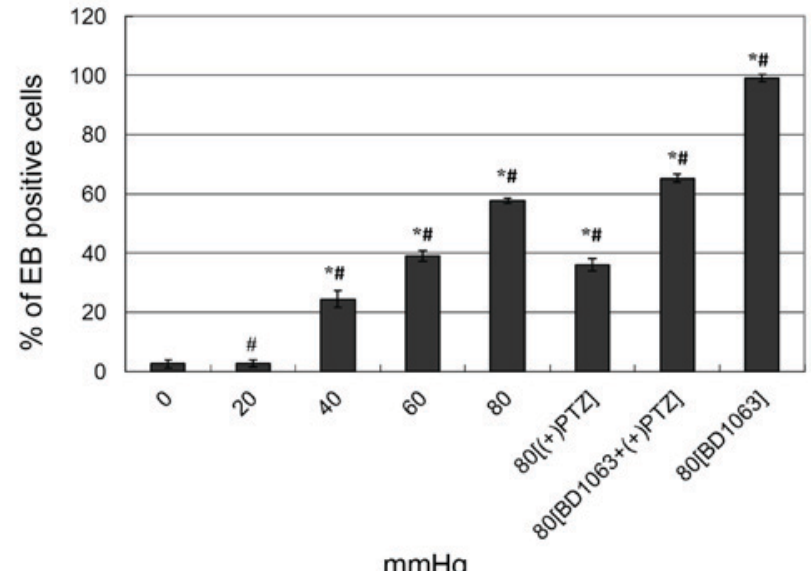

$\mathrm{mmHg}$

Figure 2. Pressure-induced apoptosis and death of hTMCs by EB/AO dual staining assay. Fluorescence images of cells captured under the microscope (x200 magnification), normal cells were stained green (AO positive), advanced apoptotic and dead cells were stained orange-red (EB positive). (Aa-e) Pressure of 0 , 20, 40, 60 and $80 \mathrm{mmHg}$, respectively, for $48 \mathrm{~h}$. (A-f) $80 \mathrm{mmHg}$ plus (+)-PTZ (20 $\mu \mathrm{M})$ at $44 \mathrm{~h}$. (A-g) $80 \mathrm{mmHg}$ plus BD-1063 (20 $\mu \mathrm{M}) 30 \mathrm{~min}$ prior to (+)-PTZ at $44 \mathrm{~h}$. (A-h) $80 \mathrm{mmHg}$ plus BD-1063 $(20 \mu \mathrm{M})$ at $44 \mathrm{~h}$ treated cells. (B) Summary of pressure-induced cell apoptosis and death. The quantitative data collected from the fluorescence images are expressed as the mean percentage \pm standard error of the mean of the ratio of apoptotic and dead cells to total cells of three different fields of cells, where each field contained 6-150 cells. ${ }^{*} \mathrm{P}<0.0001$ vs. control $\left(0 \mathrm{mmHg}\right.$ pressure); ${ }^{*} \mathrm{P}<0.0001$ vs. each other group. hTMCs, human trabecular meshwork cells; EB/AO, ethidium bromide/acridine orange; PTZ, pentazocin; BD-1063, N-(2-(3,4-dichlorophenyl)ethyl)-N-methyl-2-(dimethyla mino) ethylamine.
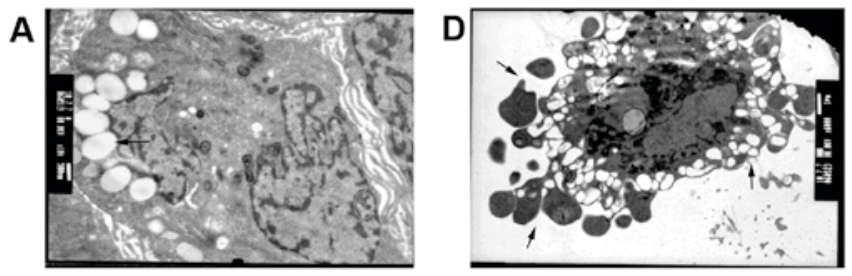

B
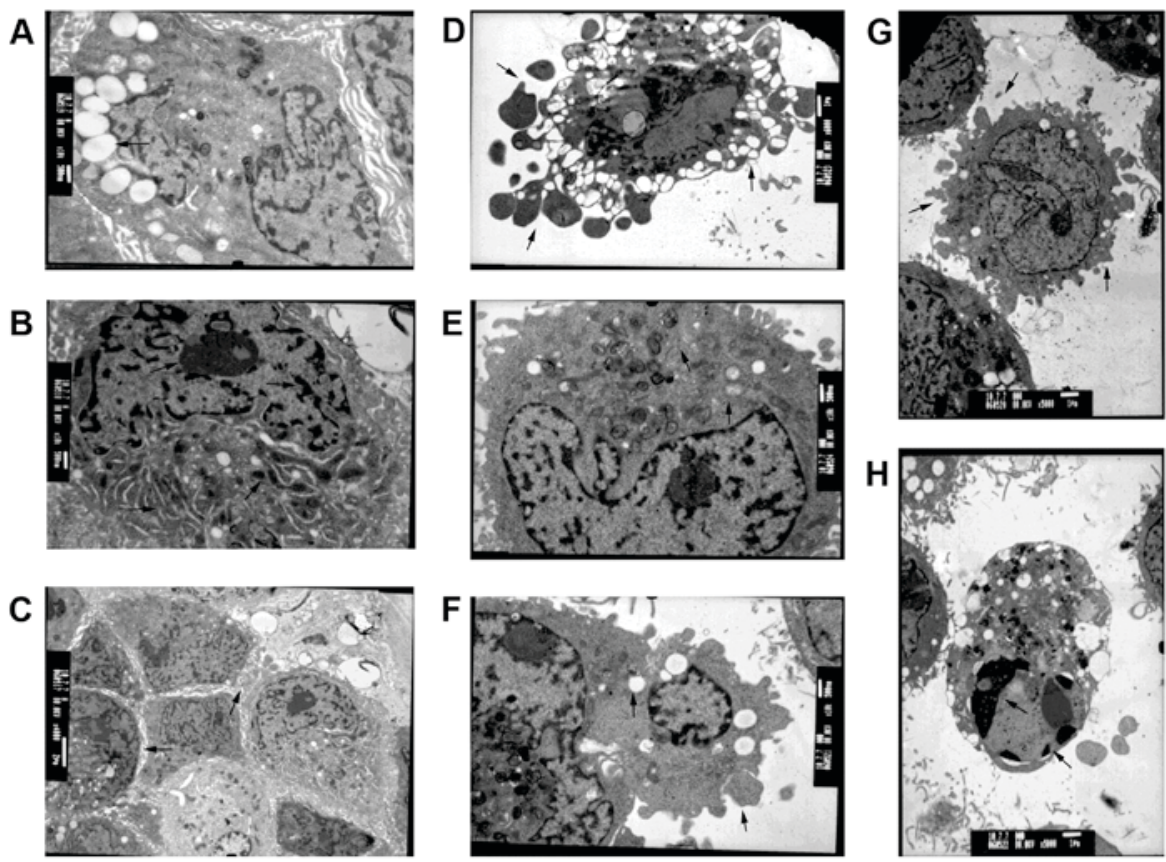

Figure 3. Pressure-induced morphologic changes of hTMCs by transmission electron microscopy. (A-C) Feature of normal hTMCs is shown. (A) Arrows indicate numerous pinosomes in hTMCs. (B) Arrows indicate abundant organelles (including mitochondria, rough endoplasmic reticulum and Golgi bodies) and a prominent spiked band of heterochromatin present along the nuclear circumference of hTMCs. (C) Arrows indicate multiple cell villous projections at cell surfaces. (D-H) Changes of $80 \mathrm{mmHg}$ pressure treated hTMCs are shown. (D) Advanced stage of apoptosis; arrows indicate abundant apoptotic bodies. (E) Swelling and disappearance of mitochondrial cristae and reduction of endoplasmic reticulum and pinosomes indicated by arrows. Arrows indicate (F) the disappearance of villous projections, reduction of pinosomes and formation of apoptotic bodies, (G) the non-age apoptosis of hTMCs; the formation of apoptotic bodies and $(\mathrm{H})$ indicates another form of non-age apoptosis; aggregation of chromatins and karyopyknosis. hTMCs, human trabecular meshwork cells. 


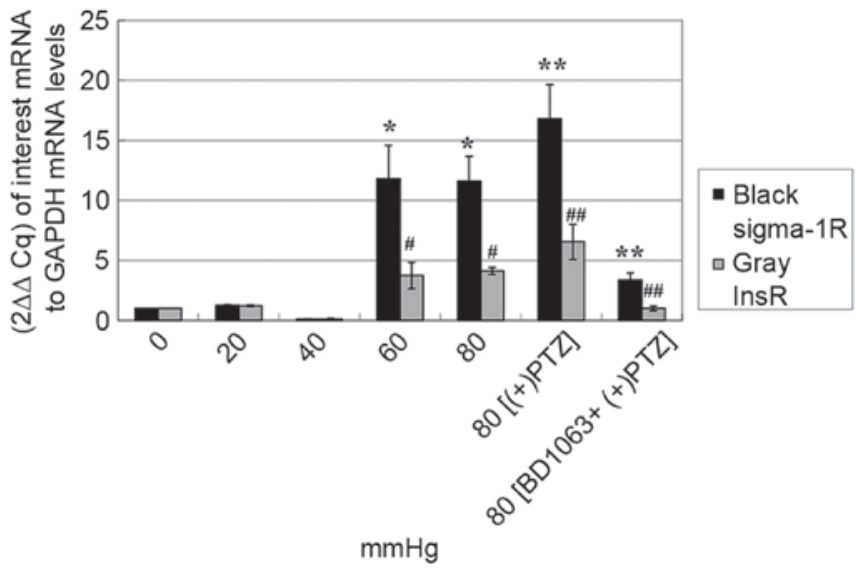

Figure 4. Pressure-induced change of Sig-1R and InsR mRNA expression in hTMCs. Total RNA was isolated from cells of each group and were subjected to RT-qPCR analysis. Cycle threshold values of Sig-1R and InsR were normalized to $\beta$-actin for each sample and calculated by comparison of $2^{\Delta \Delta C q}$ in three separate experiments. Sig-1R and InsR mRNA were increased under the pressure of 60 and $80 \mathrm{mmHg},(+)-\mathrm{PTZ}$ upregulated the Sig-1R and InsR mRNA levels of the $80 \mathrm{mmHg}$ group, while these upregulations were reversed by BD-1063. Data are presented as the mean \pm standard deviation; ${ }^{*} \mathrm{P}<0.0001$ and ${ }^{\#} \mathrm{P}<0.0001$ vs. controls $(0 \mathrm{mmHg}) ;{ }^{* *} \mathrm{P}<0.0001$ and ${ }^{\# \#} \mathrm{P}<0.0001$ vs. $80 \mathrm{mmHg}$ treated cells and $80 \mathrm{mmHg}+(+)$ PTZ and $80 \mathrm{mmHg}+\mathrm{BD} 1063+(+) \mathrm{PTZ})$ cells. Sig-1R, $\sigma-1$ receptor; InsR, insulin receptor; hTMCs, human trabecular meshwork cells; PTZ, pentazocin; BD-1063, N-(2-(3,4-dichlorophenyl)ethyl)-N-methyl-2-(dim ethylamino) ethylamine.

mediated by Sig-1R activation, the $80 \mathrm{mmHg}$-treated cells were exposed to BD-1063 prior to (+)-PTZ, which apparently increased cell apoptosis and death $(65.30 \pm 1.48, \mathrm{P}<0.0001$; Fig. 2A-g) compared with the (+)-PTZ group. These data suggested that $\mathrm{BD}-1063$ was able to block the protective effect of (+)-PTZ. The $80 \mathrm{mmHg}$-treated cells that were exposed only to BD-1063 experienced almost total apoptosis and death $(99.07 \pm 1.07, \mathrm{P}<0.0001$; Fig. 2A-h) compared with the $80 \mathrm{mmHg}$-treated cells, which suggested that antagonism of Sig-1R can cause pressure associated with cell death. In Fig. 2B, data are presented as the mean percentage \pm standard error of the mean in three fields of cells, where each field contained $60-150$ cells $(\mathrm{P}<0.0001$ between each two groups with the exception of the 0 and $20 \mathrm{mmHg}$ groups).

Pressure-induced morphologic changes of hTMCs were observed by transmission electron microscopy. Normal hTMCs exhibited multiple cell villous projections at cell surfaces, abundant organelles (including mitochondria, rough ER and Golgi bodies), numerous pinosomes, clear nucleoli and prominent spiked bands of heterochromatin along the nuclear circumference (Fig. 3A-C). The ultra-microstructural changes observed in the $80 \mathrm{mmHg}$-treated group were the formation of apoptotic bodies, swelling and disappearance of mitochondrial cristae, reduction of ER and pinosomes, aggregation of chromatins and karyopyknosis (Fig. 3D-H). The images indicated that high pressure-treated cells were undergoing an apoptotic procedure.

Pressure-induced change of Sig-1R and InsR $m R N A$ expression in hTMCs. RT-qPCR analysis of Sig-1R mRNA expression of seven groups of cells [0, 20, 40, 60 and $80 \mathrm{mmHg}$ for $48 \mathrm{~h}$, respectively, $80 \mathrm{mmHg}$ plus (+)-PTZ $(20 \mu \mathrm{M})$ at the $44 \mathrm{~h}$, $80 \mathrm{mmHg}$ plus BD-1063 $(20 \mu \mathrm{M}) 30 \mathrm{~min}$ prior to (+)-PTZ at

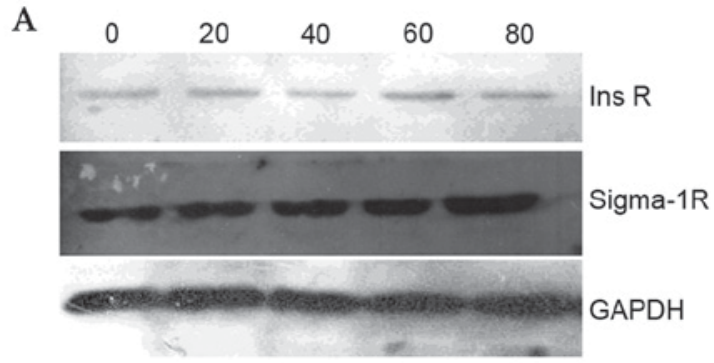

B

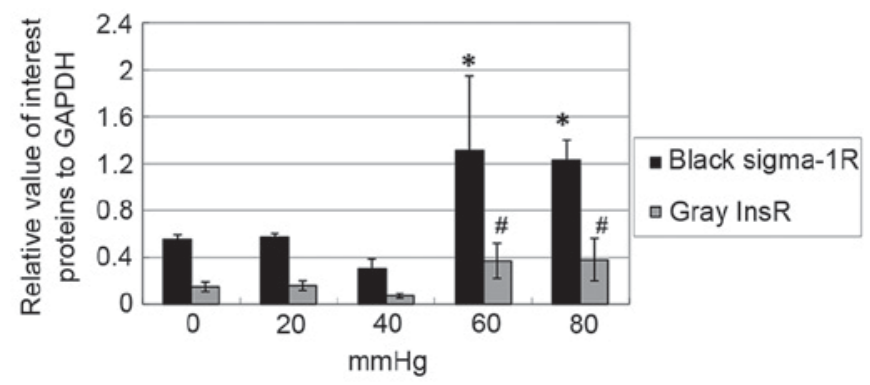

Figure 5. Pressure-induced change $(0-80 \mathrm{mmHg})$ of Sig-1R and InsR protein expression in hTMCs. (A) Western blotting: The molecular sizes of the Sig-1R, InsR and GAPDH bands of $0-80 \mathrm{mmHg}$ groups are indicated. (B) Data from densitometric scans of blots in (A) indicate that Sig-1R and InsR proteins of 60 and $80 \mathrm{mmHg}$ groups were increased compared with that of 0,20 and $40 \mathrm{mmHg}$ groups. Data are presented as the mean \pm standard deviation; ${ }^{*} \mathrm{P}=0.0068$ and ${ }^{\#} \mathrm{P}=0.0177 \mathrm{vs}$. controls $(0 \mathrm{mmHg})$. Sig-1R, o-1 receptor; InsR, insulin receptor; hTMCs, human trabecular meshwork cells.

the $44 \mathrm{~h}$ ] demonstrated that the amount of Sig-1R under pressures of 60 and $80 \mathrm{mmHg}$ were 11- to 12-fold higher compared with the 0,20 and $40 \mathrm{mmHg}$ groups; (+)-PTZ upregulated the Sig-1R mRNA levels of $80 \mathrm{mmHg}$ group, while the upregulation was reversed by BD-1063 ( $\mathrm{P}<0.0001$; Fig. 4). The results suggested that short-term exposure to high pressure can cause upregulation of Sig-1R mRNA expression in vitro.

The expression of InsR mRNA in the seven groups of cells was consistent with that of Sig-1R; the amount of InsR under pressures of 60 and $80 \mathrm{mmHg}$ were 3-to 4-fold higher compared with the 0,20 and $40 \mathrm{mmHg}$ groups. Furthermore, the change in InsR mRNA levels coincided with those of Sig-1R when administering (+)-PTZ/BD-1063 ( $\mathrm{P}<0.0001$; Fig. 4).

Pressure-induced change of Sig-1R, InsR and p/t ERK protein expression in hTMCs. Western blot analysis of Sig-1R and InsR protein (the seven groups of cells as with RT-qPCR analysis above) were consistent with the result of RT-qPCR ( $<<0.05$; Fig. 5A). This indicated that high pressure can also cause the upregulations of Sig-1R and InsR protein expression in vitro. To explore the association between Sig-1R and InsR signaling, the phosphorylation of ERK (an important downstream protein of the InsR-MAPK signal pathway) was analyzed by western blotting. The data demonstrated that (+)-PTZ caused an increase of Sig-1R itself, InsR and pERK protein expression ( $\mathrm{P}<0.05$; Fig. 6; tERK was unchanged). To confirm whether this effect was mediated by Sig-1R, BD-1063 was used $30 \mathrm{~min}$ prior to (+)-PTZ and the results demonstrated that the effect was attenuated by BD-1063 (P<0.05; Fig. 6). It was notable that 


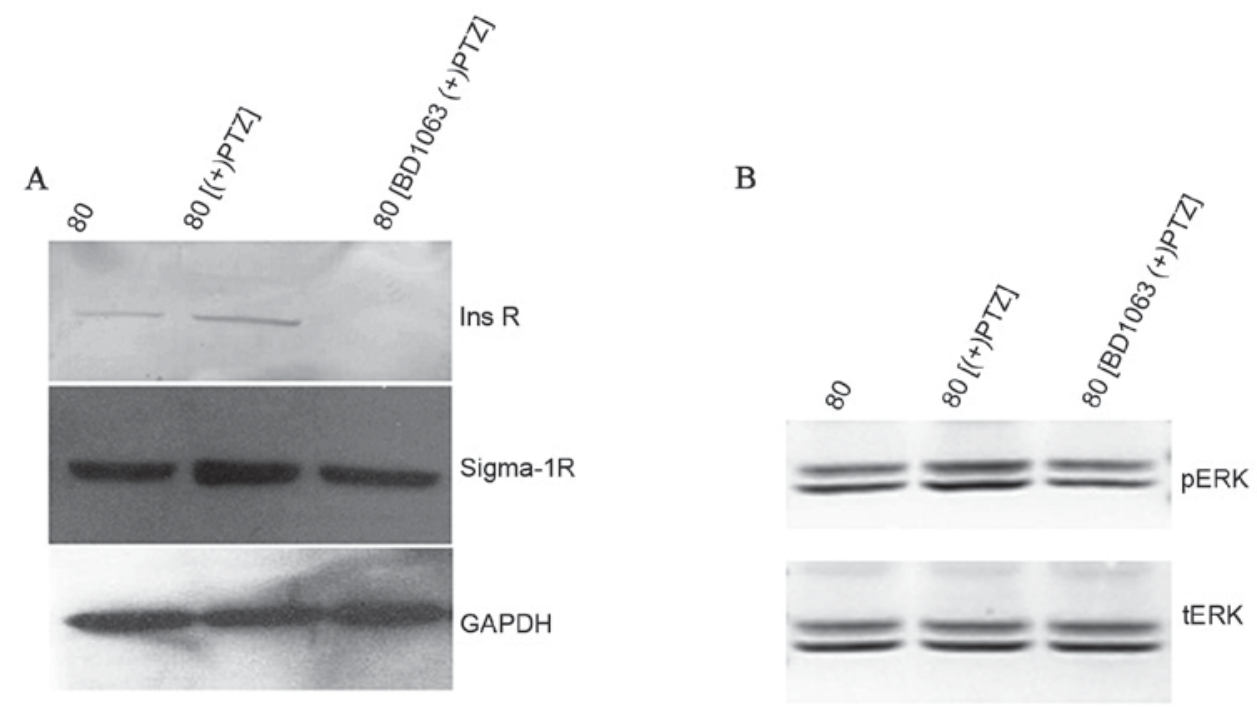

$\mathrm{C}$

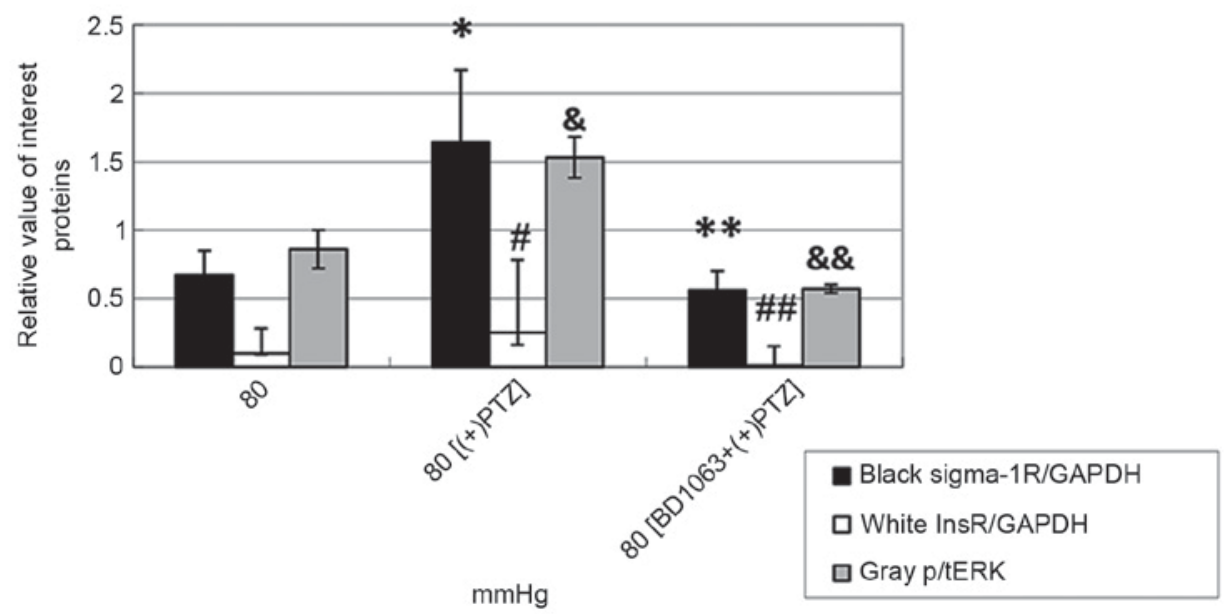

Figure 6. Expression of Sig-1R, InsR and p/tERK1/2 protein in hTMCs treated with (+)PTZ and BD-1063. (A) Western blotting: The molecular sizes of the Sig-1R, InsR and GAPDH bands of $80 \mathrm{mmHg}, 80 \mathrm{mmHg}+(+) \mathrm{PTZ}$, and $80 \mathrm{mmHg}+\mathrm{BD}-1063+(+)$ PTZ groups are indicated. (B) Western blotting: The molecular sizes of the pERK1/2 and tERK1/2 bands of $80 \mathrm{mmHg}, 80 \mathrm{mmHg}+(+) \mathrm{PTZ}$, and $80 \mathrm{mmHg}+\mathrm{BD}-1063+(+) \mathrm{PTZ}$ groups are indicated. (C) Data from densitometric scans of blots from (A and B); Sig-1R and InsR proteins were increased simultaneously when (+)PTZ was administered, and this effect was attenuated by BD-1063. Data are presented as the mean \pm standard deviation. ${ }^{*} \mathrm{P}=0.0002$ and ${ }^{\#} \mathrm{P}=0.0039$ vs. controls $(80 \mathrm{mmHg}){ }^{* * *} \mathrm{P}=0.0002$ and ${ }^{\# \#} \mathrm{P}=0.0039$ vs. $80 \mathrm{mmHg}+(+) \mathrm{PTZ}$ group. p/tERK1/2 was increased when (+)PTZ was administered and such effect was also attenuated by BD-1063. \&P=0.0137 vs. control $(0 \mathrm{mmHg}) ;{ }^{\&} \mathrm{P}=0.0137$ vs. $80 \mathrm{mmHg}+(+) \mathrm{PTZ}$ group. Data are presented as the mean \pm standard deviation. Sig-1R, $\sigma-1 \mathrm{receptor}$; InsR, insulin receptor; p/t, phosphorylated and total; ERK, extracellular signal-regulated kinase; hTMCs, human trabecular meshwork cells; PTZ, pentazocin; BD-1063, N-(2-(3,4-dichlorophenyl)ethyl)-N-methyl-2-(dimethylamino) ethylamine Sig-1R, o-1 receptor; InsR, insulin receptor.

Sig-1R ligands modulated InsR and pERK expression, which indicated that Sig-1R served its functions partly by activating InsR and its MAPK signal pathway.

\section{Discussion}

A previous study demonstrated that Sig-1R agonist can decrease IOP and protect against retinal damage in a rat model of chronic ocular hypertension glaucoma (26). Numerous researchers (29-31) have performed experiments to study the anti-apoptotic mechanism of Sig-1R agonist on RGCs, however, the mechanism of IOP lowering remains to be fully elucidated. The present study identified that the Sig-1R agonist (+)-PTZ protects hTMCs from pressure-induced apoptosis and death by activating InsR and its MAPK pathway.

Elevated IOP is the main risk factor for the development and progression of glaucomatous damage $(32,33)$. It is hypothesized that elevated IOP results from increased aqueous outflow resistance and is due to several morphologic changes in the TM, including loss of TMCs $(34,35)$. As a result, it was considered useful to observe what TMCs experience under the high air-pressure directly. In the present study, hTMCs were cultured under high pressure $(80 \mathrm{mmHg})$ and it was observed that the cells underwent an apoptotic process, with $50 \%$ cell death at $48 \mathrm{~h}$. A loss of the hTMCs, followed by substitution with extracellular matrix (ECM), may contribute to an increased resistance to $\mathrm{AH}$ outflow $(36,37)$. Morphologic changes were also observed in hTMCs cultured under high pressure. Among the abnormalities, the reduction of pinosomes was notable and possibly meant reduced phagocytic activity in glaucomatous TMCs, which would induce increased deposition of ECM material in the outflow pathway (38). Previous studies also suggested that these structural changes in the TM and a decrease in TMCs are also triggered by apoptosis $(35,39,40)$. These hypotheses, together with the findings of the present study, indicate that TMCs serve a key role in maintaining the 
balance of drainage pathways, that air-pressure over $40 \mathrm{mmHg}$ can cause a decrease in hTMCs and a disequilibrium between the cells and ECM and that such disequilibrium can concomitantly cause increased IOP. As a result, finding a way to protect hTMCs from apoptosis and death is important.

Sig-1R has been studied thoroughly in CNS, where it acts as a neuroprotector. In the eye, Sig-1Rs are located in the lacrimal glands, retina, iris-ciliary body, cornea and lens, and the effects of modulating their activities include lowering IOP and protecting RGCs against stress (15-17). Bucolo et al (22) demonstrated that topical agonists [(+)-PTZ and flunarizine] ) caused a significant dose-related reduction of IOP in ocular normotensive and hypertensive albino rabbits. A previous study also demonstrated that intraperitoneal injection of a Sig-1R agonist (pregnenolone) reduced IOP in a chronic ocular hypertension rat model (27). It was hypothesized that this may be caused by the increasing $\mathrm{AH}$ outflow through the other quadrants of TM with normal episcleral vein pressure. Thus, it was required to perform in vitro experiments to explain the phenomenon. The present study demonstrated that air-pressure over $40 \mathrm{mmHg}$ may contribute to the apoptosis and death of hTMCs. The Sig-1R agonist (+)-PTZ can prevent the process of apoptosis and maintain the quantity and structure of these cells, which is beneficial for the homeostasis of TM. A short time exposure to high air-pressure of hTMCs caused an increase of Sig-1Rs, which may be beneficial to the survival of the cells. This result corresponds to the hypothesis that, besides ligands, Sig-1Rs can also response for cellular stress, including deprivation of glucose, deplenishment of calcium in ER and oxidation (24); high pressure being a type of stress.

For years, an increasing number of epidemiological studies demonstrated that patients with diabetes exhibit an increased risk of developing POAG $(15,41-44)$. A possible reason for the impairment of InsRs in patients with diabetes inducing InsR signaling dysfunction, may result in decreased phosphorylation of protein kinase B and ERK1/2. This decrease may cause apoptosis and death of hTMCs, unbalanced homeostasis of $\mathrm{TM}$ and an increase in the resistance of $\mathrm{AH}$ outflows. The present study also observed that the expression of InsR was highly consistent with that of Sig-1R. Sig-1R agonist (+)-PTZ upregulated the expression of Sig-1R and InsR at $80 \mathrm{mmHg}$ and that can be attenuated by the antagonist BD-1063. To evaluate whether the upregulation of InsR triggered downstream cascade, the phosphorylation of ERK1/2, a protein of InsR-MAPK signaling pathway, was also detected. The phosphorylation of ERK1/2 increased with the upregulation of InsR when (+)-PTZ was administered to $80 \mathrm{mmHg}$-cultured cells, and this effect can be attenuated by BD-1063. These results led to the hypothesis that the anti-apoptotic mechanism of Sig-1R on pressure-induced damage of hTMCs is associated with the MAPK signal pathway. The current consensus is that the acute metabolic effects of insulin require activation of the IRS-PI3K pathway, while stimulation of cell growth and proliferation requires the Ras-MAPK cascade (45).

Previous studies have demonstrated that the Sig-1R is mainly located at the MAM (46-48). Activation and cellular stress promote redistribution of the receptors from MAM to other subcellular locations, including the periphery of endoplasmic membranes, the vicinity of the cell membrane or nuclear envelopes. This relocation possibly increases the number or types of proteins with which the Sig-1R can interact. The results from the current study may provide a new protein, InsR, interacting with the Sig-1R, a hypothesis which requires further elucidation.

In conclusion, the present study demonstrates that high pressure can induce hTMCs apoptosis and death, which is one of the causes of the progression of POAG. The finding that the Sig-1R agonist (+)-PTZ protects hTMCs from pressure-induced apoptosis and death by activating InsR and the MAPK signal pathway, together with the retina protecting effect of the receptor, may make the Sig-1R agonist a superior therapeutic for POAG in the near future.

\section{Acknowledgements}

The present study was supported by the Youth Scientific Funds of Heilongjiang Province of China (grant no. QC2014C089), the Doctoral Foundation of Ministry of Education of China (grant no. 20112307110015) and the Sub-project of National 973 Foundation (grant no. 2100CB707502).

\section{References}

1. Quigley HA, Tielsch JM, Katz J and Sommer A: Rate of progression in open-angle glaucoma estimated from cross-sectional prevalence of visual field damage. Am J Ophthalmol 122: 355-363, 1996.

2. Harper MM, Adamson L, Blits B, Bunge MB, Grozdanic SD and Sakaguchi DS: Brain-derived neurotrophic factor released from engineered mesenchymal stem cells attenuates glutamate and hydrogen peroxide-mediated death of staurosporine-differentiated RGC-5 cells. Exp Eye Res 89: 538-548, 2006.

3. Boland MV and Quigley HA: Risk factors and open-angle glaucoma: Classification and application. J Glaucoma 16: 406-418, 2007.

4. Cruz-lñigo Y, Izquierdo NJ, García O and Pérez R: Open-angle glaucoma in patients with diabetic retinopathy at the Puerto Rico Medical Center. Bol Asoc Med P R 104: 10-13, 2012.

5. Chopra V, Varma R, Francis BA, Wu J, Torres M and Azen SP. Los Angeles Latino Eye Study Group: Type 2 diabetes mellitus and the risk of open-angle glaucoma the Los Angeles Latino Eye Study. Ophthalmology 115: 227-232.e1, 2008.

6. Mitchell P, Smith W, Chey T and Henley P: Open-angle glaucoma and diabetes: The Blue Mountains Eye Study, Australia. Ophthalmology 104: 712-718, 1997.

7. Sato T and Roy S: Effect of high glucose on fibronectin expression and cell proliferation in trabecular meshwork cells. Invest Ophthalmol Vis Sci 43: 170-175, 2002.

8. Nakamura M,Kanamori A and Negi A: Diabetes mellitus as a risk factor for glaucomatous optic neuropathy. Ophthalmologica 219: $1-10,2005$.

9. Yang Q, Xu Y, Xie P, Cheng H, Song Q, Su T, Yuan S and Liu Q: Retinal neurodegeneration in $\mathrm{db} / \mathrm{db}$ mice at the early period of diabetes. J Ophthalmol 2015: 757412, 2015.

10. Yang Y, Mao D, Chen X, Zhao L, Tian Q, Liu C and Zhou BL: Decrease in retinal neuronal cells in streptozotocin-induced diabetic mice. Mol Vis 18: 1411-1420, 2012.

11. de Moraes $\mathrm{G}$ and Layton CJ: Therapeutic targeting of diabetic retinal neuropathy as a strategy in preventing diabetic retinopathy. Clin Exp Ophthalmol 44: 838-852, 2016.

12. Soto I, Howell GR, John CW, Kief JL, Libby RT and John SW: DBA/2J mice are susceptible to diabetic nephropathy and diabetic exacerbation of IOP elevation. PLoS One 9: e107291, 2014.

13. Wong VH, Bui BV and Vingrys AJ: Clinical and experimental links between diabetes and glaucoma. Clin Exp Optom 94: 4-23, 2011.

14. Faiq MA, Dada R, Saluja D and Dada T: Glaucoma-diabetes of the brain: A radical hypothesis about its nature and pathogenesis. Med Hypotheses 82: 535-546, 2014.

15. Shen L, Walter S, Melles RB, Glymour MM and Jorgenson E: Diabetes pathology and risk of primary open-angle glaucoma: Evaluating causal mechanisms by using genetic information. Am J Epidemiol 183: 147-155, 2016. 
16. Nakae J, Kido Y and Accili D: Distinct and overlapping functions of insulin and IGF-I receptors. Endocr Rev 22: 818-835, 2001.

17. Baumann CA and Saltiel AR: Spatial compartmentalization of signal transduction in insulin action. Bioessays 23: 215-222, 2001.

18. Saltiel AR and Kahn CR: Insulin signalling and the regulation of glucose and lipid metabolism. Nature 414: 799-806, 2001.

19. Johnston AM, Pirola L and Van Obberghen E: Molecular mechanisms of insulin receptor substrate protein-mediated modulation of insulin signalling. FEBS Lett 546: 32-36, 2003.

20. Ola MS, Moore P, El-Sherbeny A, Roon P, Agarwal N, Sarthy VP, Casellas P, Ganapathy V and Smith SB: Expression pattern of sigma receptor $1 \mathrm{mRNA}$ and protein in mammalian retina. Brain Res Mol Brain Res 95: 86-95, 2001.

21. Campana G, Bucolo C, Murari G and Spampinato S: Ocular hypotensive action of topical flunarizine in the rabbit: Role of sigma 1 recognition sites. J Pharmacol Exp Ther 303: 1086-1094, 2002.

22. Bucolo C, Campana G, Di Toro R, Cacciaguerra S and Spampinato S: Sigma1 recognition sites in rabbit iris-ciliary body: Topical sigma1-site agonists lower intraocular pressure. J Pharmacol Exp Ther 289: 1362-1369, 1999.

23. Martin PM, Ola MS, Agarwal N, Ganapathy V and Smith SB: The sigma receptor ligand (+)-pentazocine prevents apoptotic retinal ganglion cell death induced in vitro by homocysteine and glutamate. Brain Res Mol Brain Res 123: 66-75, 2004.

24. Hayashi T and Su TP: Sigma-1 receptor chaperones at the ER-mitochondrion interface regulate $\mathrm{Ca}(2+)$ signaling and cell survival. Cell 131: 596-610, 2007.

25. Su TP and Hayashi T: Understanding the molecular mechanism of sigma-1 receptors: Towards a hypothesis that sigma-1 receptors are intracellular amplifiers for signal transduction. Curr Med Chem 10: 2073-2080, 2003.

26. Cobos EJ, Entrena JM, Nieto FR, Cendán CM and Del Pozo E: Pharmacology and therapeutic potential of sigma(1) receptor ligands. Curr Neuropharmacol 6: 344-366, 2009.

27. Sun X, Cheng F, Meng B, Yang B, Song W and Yuan H: Pregnenolone sulfate decreases intraocular pressure and changes expression of sigma receptor in a model of chronic ocular hypertension. Mol Biol Rep 39: 6607-6614, 2012.

28. Livak KJ and Schmittgen TD: Analysis of relative gene expression data using real-time quantitative PCR and the 2(-Delta DeltaC(T)) method. Methods 25: 402-408, 2001.

29. Zhao J, Mysona BA, Qureshi A, Kim L, Fields T, Gonsalvez GB, Smith SB and Bollinger KE: (+)-Pentazocine reduces NMDA-induced murine retinal ganglion cell death through a 61 -dependent mechanism. Invest Ophthalmol Vis Sci 57: 453-461, 2016

30. Mueller BH II, Park Y, Ma HY, Dibas A, Ellis DZ, Clark AF and Yorio T: Sigma-1 receptor stimulation protects retinal ganglion cells from ischemia-like insult through the activation of extracellular-signal-regulated kinases 1/2. Exp Eye Res 128: 156-169, 2014.

31. Mueller BH II, Park Y, Daudt DR III, Ma HY, Akopova I, Stankowska DL, Clark AF and Yorio T: Sigma-1 receptor stimulation attenuates calcium influx through activated L-type voltage gated calcium channels in purified retinal ganglion cells. Exp Eye Res 107: 21-31, 2013.

32. Kass MA, Heuer DK, Higginbotham EJ, Johnson CA, Keltner JL, Miller JP, Parrish RK II, Wilson MR and Gordon MO: The ocular hypertension treatment study: A randomized trial determines that topical ocular hypotensive medication delays or prevents the onset of primary open-angle glaucoma. Arch Ophthalmol 120: 701-713, 829-830, 2002.
33. Heijl A, Leske MC, Bengtsson B, Hyman L and Hussein M; Early Manifest Glaucoma Trial Group: Reduction of intraocular pressure and glaucoma progression: Results from the Early Manifest Glaucoma Trial. Arch Ophthalmol 120: 1268-1279, 2002.

34. Alvarado J, Murphy C and Juster R: Trabecular meshwork cellularity in primary open-angle glaucoma and nonglaucomatous normals. Ophthalmology 91: 564-579, 1984.

35. Rohen JW, Lütjen-Drecoll E, Flugel C, Meyer M and Grierson I: Ultrastructure of the trabecular meshwork in untreated cases of primary open-angle glaucoma (POAG). Exp Eye Res 56: 683-692, 1993.

36. Lutjen-Drecoll E, Rittig M, Rauterberg J, Jander R and Mollenhauer J: Immunomicroscopical study of type VI collagen in the trabecular meshwork of normal and glaucomatous eyes. Exp Eye Res 48: 139-147, 1989.

37. Tripathi RC: Pathologic anatomy of the outflow pathway of aqueous humor in chronic simple glaucoma. Exp Eye Res 25 (Suppl): S403-S407, 1977.

38. Zhang X, Ognibene CM, Clark AF and Yorio T: Dexamethasone inhibition of trabecular meshwork cell phagocytosis and its modulation by glucocorticoid receptor beta. Exp Eye Res 84: 275-284, 2007.

39. Cao Y, Wei H, Pfaffl M, Da B and Li Z: Apoptosis of human trabecular meshwork cells induced by transforming growth factor-beta2 in vitro. J Huazhong Univ Sci Technolog Med Sci 24: 87-89, 94, 2004.

40. Ziangirova GG and Antonova OV: Lipid peroxidation in the pathogenesis of primary open-angle glaucoma. Vestn Oftalmol 119: 54-55, 2003 (In Russian).

41. Zhao D, Cho J, Kim MH, Friedman DS and Guallar E: Diabetes, fasting glucose, and the risk of glaucoma: A metaanalysis. Ophthalmology 122: 72-78, 2015.

42. Ko F, Boland MV, Gupta P, Gadkaree SK, Vitale S, Guallar E, Zhao D and Friedman DS: Diabetes, triglyceride levels, and other risk factors for glaucoma in the National Health and Nutrition Examination Survey 2005-2008. Invest Ophthalmol Vis Sci 57: 2152-2157, 2016.

43. Mitchell P, Smith W, Chey T and Healey PR: Open-angle glaucoma and diabetes: The Blue Mountains eye study, Australia. Ophthalmology 104: 712-718, 1997.

44. Klein BE, Klein R and Jensen SC: Open-angle glaucoma and older-onset diabetes. The Beaver Dam Eye Study. Ophthalmology 101: 1173-1177, 1994.

45. Xie L, Chen H, Overbeek PA and Reneker LW: Elevated insulin signaling disrupts the growth and differentiation pattern of the mouse lens. Mol Vis 13: 397-407, 2007.

46. Zamanillo D, Romero L, Merlos M and Vela JM: Sigma 1 receptor: A new therapeutic target for pain. Eur J Pharmacol 716: 78-93, 2013.

47. Mori T, Hayashi T, Hayashi E and Su TP: Sigma-1 receptor chaperone at the ER-mitochondrion interface mediates the mitochondrion-ER-nucleus signaling for cellular survival. PLoS One 8: e76941, 2013.

48. Su TP, Hayashi T, Maurice T, Buch S and Ruoho AE: The sigma-1 receptor chaperone as an inter-organelle signaling modulator. Trends Pharmacol Sci 31: 557-566, 2010. 